\title{
Neutrophil-to-lymphocyte Ratio Prior to Each Chemotherapy Line Predicts Clinical Outcomes in Patients With Unresectable Gastric Cancer
}

Hiroaki Tanioka

Kawasaki Medical School: Kawasaki Ika Daigaku

Shuya Yano

Kawasaki Medical School: Kawasaki Ika Daigaku

Makoto Okawaki

Kawasaki Medical School: Kawasaki Ika Daigaku

Yosuke Katata

Kawasaki Medical School: Kawasaki Ika Daigaku

Tomomi Yoshimitsu

Kawasaki Medical School: Kawasaki Ika Daigaku

Kikue Tokuda

Kawasaki Medical School: Kawasaki Ika Daigaku

Akihiro Nyuya

Kawasaki Medical School: Kawasaki Ika Daigaku

Takeshi Nagasaka ( $\nabla$ takeshin@med.kawasaki-m.ac.jp)

Kawasaki Medical University https://orcid.org/0000-0002-4236-350X

\section{Research}

Keywords: Gastric cancer, Neutrophil-to-lymphocyte ratio, Biomarker, Chemotherapy, Nivolumab

Posted Date: October 20th, 2021

DOI: https://doi.org/10.21203/rs.3.rs-960283/v1

License: (c) (i) This work is licensed under a Creative Commons Attribution 4.0 International License. Read Full License 


\section{Abstract}

Background: The neutrophil-to-lymphocyte ratio (NLR) is a well-known prognostic marker for gastric cancer (GC) patients. However, for GC patients treated with palliative chemotherapy, the prognostic value of NLR remains obscure. Therefore, we evaluated the clinical impact of NLR in GC patients treated with multiple lines of chemotherapy.

Methods: We retrospectively evaluated 83 unresectable GC patients who received a series of chemotherapy treatments. NLR in the blood was calculated before the initiation of each line of chemotherapy (before 1st-, 2nd-, and 3rd-line, respectively).

Results: Of the 83 patients enrolled, 56 (67\%) received 2nd-line chemotherapy and 34 (41\%) received 3rdline treatment. NLR at 1 st-line treatment ranged from 0.72 to 48.9 (the median NLR was 3.00). Thus, we used this median NLR value (3.00) as the cut-off for NLR throughout this study. All patients were divided into a NLR-high $(\geq 3.00$ ) or NLR-low group $(<3.00)$ by NLR values determined at before each line of chemotherapy. The median overall survival (OS) of the NLR-low group was better than that of the NLRhigh group throughout $1 \mathrm{st}$ - to $3 \mathrm{rd}$-line treatment (1st-line: 18.1 vs. 8.0 months, $p=0.06$; 2 nd-line: 10.7 vs. 4.5 months, $p=0.0001$; 3rd-line: 8.7 vs. 4.7 months, $p=0.003$ ), respectively. Of the 34 patients who received 3rd-line chemotherapy, 24 were treated with nivolumab. Of the 24 patients treated with nivolumab, patients in the NLR-low group showed better OS compared with those in the NLR-high group (8.3 months in NLR-low and 6.6 months in NLR-high, $p=0.06$ ).

Conclusions: Estimates of NLR prior to each chemotherapy line may be readily performed in a clinical setting and are useful for predicting outcome in unresectable GC patients, including in patients treated with nivolumab.

\section{Background}

Gastric cancer (GC) is the fifth common cancer and the third common cause of cancer-related mortality worldwide (1). Systemic chemotherapy including cytotoxic agents, molecular-targeted drugs, or immune checkpoint inhibitors has demonstrated a statistically significant prolongation of survival compared with best supportive care alone. Therefore, it is recommended as first-line or later conventional treatment for advanced GC (2-4). In addition to other cancers, blockade of program death-1 (PD-1) or program death ligand-1 (PD-L1) restores T-cell activity and has emerged as a breakthrough therapy for GC.

Among the specific drugs, nivolumab, a PD-1 inhibitor, was evaluated for its efficacy against GC in a randomized phase III study, ATTRACTION-2 (5). The ATTRACTION-2 results indicated that patients treated with nivolumab exhibited significantly prolonged OS compared with those treated with placebo. Although this represents an advancement in GC treatment, the outcome of patients with unresectable GC remains poor. Indeed, the median OS is approximately 1 year after initiation of first-line chemotherapy (6). In the ATTRACTION-2 study, the patients receiving nivolumab showed a significant increase in median OS, 
however, approximately half experienced exhibited progressive disease at their first radiographical examination. Thus, biomarkers which can predict outcome or efficacy of these agents are needed.

Systemic inflammation plays an important role in tumor promotion and progression (7). Several markers of systemic inflammation including $C$ reactive protein, platelet-to-lymphocyte ratio, and NLR are associated with clinical outcome in various cancers. The peripheral blood NLR, an indicator of systemic inflammation, is a conventional biomarker in clinical the setting, and has been reported as a prognostic biomarker in solid neoplasms, such as breast, lung, pancreas, colon, rectum, and stomach (8-12). In addition to its prognostic role, NLR has been recognized as a predictive marker for several unresectable solid neoplasm treatments with cytotoxic chemotherapy and immunotherapy $(13,14)$. Recently, Valero et al. demonstrated that increased NLR is significantly associated with poorer OS and progression-free survival, and lower response rates and clinical benefit after immune checkpoint inhibitor (ICI) therapy for multiple cancer types in a retrospective cohort of 1,714 patients representing 16 different cancer types, including GC patients treated with ICl (15).

Although the NLR values fluctuate over time, most studies have used NLR values obtained at one time point prior to the induction of targeted agent therapy to evaluate clinical outcome. In this study, we evaluated NLR values in blood at a point before the initiation of each chemotherapy line (e.g., before 1st-, 2nd-, and 3rd-line, respectively) to determine whether pretreatment NLR could predict clinical outcome.

\section{Methods}

\section{The aim of this study}

The aim of this study was to determine whether NLR values in blood at the point before the initiation of each line of chemotherapy (before 1st-, 2nd-, and 3rd-line, respectively) could predict clinical outcome in GC.

\section{Patients}

In this retrospective study, we analyzed 83 patients with unresectable GC who received palliative chemotherapy in the Department of Clinical Oncology, Kawasaki Medical School Hospital between the period of March 2018 and December 2020. All patients received one or more lines of chemotherapy and at least one evaluation of anti-tumor efficacy by computed tomography after the start of chemotherapy. The duration of follow-up ranged from 1.0 to 58.4 months (median: 10.1 months). OS was calculated from the initiation of each line of chemotherapy to death.

\section{Chemotherapy}

All patients received 1st-line chemotherapy (e.g., SOX, XELOX, FOLFOX SP, XP, weekly sb-paclitaxel plus ramucirumab, weekly nab-paclitaxel plus ramucirumab, or docetaxel monotherapy) according to the Japanese GC treatment guidelines. Details of the number of patients using each regimen are presented in Table 1. These regimens were administered as follows: 1) SOX: twice daily for the first 2 weeks of a 3- 
week cycle of S-1 (80-120 mg/day) with $100 \mathrm{mg} / \mathrm{m}^{2}$ of oxaliplatin on day one; 2) XELOX: twice daily for the first 2 weeks of a 3-week cycle of capecitabine ( 2400 to $4200 \mathrm{mg} /$ day) with $130 \mathrm{mg} / \mathrm{m}^{2}$ of oxaliplatin on day one; 3) FOLFOX: oxaliplatin $85 \mathrm{mg} / \mathrm{m}^{2}$ and LV $200 \mathrm{mg} / \mathrm{m}^{2}$ followed by bolus 5 -FU $400 \mathrm{mg} / \mathrm{m}^{2}$ and continuous 5-FU $2,400 \mathrm{mg} / \mathrm{m}^{2}$ were intravenously infused every 2 weeks; 4) SP regimen: twice daily for the first 3 weeks of a 5-week cycle of S-1 (80-120 mg/day) with $60 \mathrm{mg} / \mathrm{m}^{2}$ of cisplatin on day 8 of each cycle; 5) XP regimen: twice daily for the first 2 weeks of a 3-week cycle of capecitabine (2400-4200 $\mathrm{mg} /$ day) with $100 \mathrm{mg} / \mathrm{m}^{2}$ of cisplatin on day one; 6) Sb-paclitaxel plus ramucirumab regimen: ramucirumab (8mg/kg intravenously on days 1 and 15) with sb-paclitaxel $\left(80 \mathrm{mg} / \mathrm{m}^{2}\right.$ intravenously on days 1,8 , and 15) every 4 weeks. 7) Nab-paclitaxel plus ramucirumab regimen: ramucirumab $(8 \mathrm{mg} / \mathrm{kg}$ intravenously on days 1 and 15$)$ with nab-paclitaxel $\left(100 \mathrm{mg} / \mathrm{m}^{2}\right.$ intravenously on days 1,8 , and 15$)$ every 4 weeks; and 8) Docetaxel regimen: docetaxel $\left(60-70 \mathrm{mg} / \mathrm{m}^{2}\right)$ was administered intravenously on day 1 every 3 weeks.

Dose reduction and/or cycle delays were permitted according to the decision of each physician. Fifteen patients that were HER2-positive received tratuzumab in combination with the SOX or XP regimen.

\section{Evaluation of NLR in the blood}

NLR was calculated by dividing the absolute neutrophil and lymphocyte counts measured in peripheral blood prior to each line of chemotherapy. We used the median value of NLR obtained before 1 st-line treatment initiation as a definite cut-off value for NLR throughout the study. Patients were divided into an NLR-high group ( $\geq$ cut-off value) and an NLR-low group (< cut-off value) based on the NLR value determined before the initiation of each line of chemotherapy.

\section{Statistical analyses}

The primary aim was to evaluate the association between peripheral blood NLR and clinical outcome in GC patients. OS was calculated using the Kaplan-Meier method and the log-rank test was used to compare survival between groups. Fisher's exact test was performed to compare clinical characteristics between two groups and the Wilcoxon rank sum test was applied to continuous valuables in the case of two groups. All statistical tests were two-sided and p-values $<0.05$ were considered statistically significant. All statistical analyses were performed with EZR software (Saitama Medical Centre, Jichi Medical University, Saitama, Japan, version 1.40), which is a graphical user interface for R (The R Foundation for Statistical Computing, Vienna, Austria, version 3.5.2) [15].

\section{Results}

\section{Patient characteristics}

A total of 83 patients were enrolled. Patient characteristics are shown in Table 1. The median age was 72 (range: $44-86)$ and $63 \%(n=52)$ of the patients were male. Fifty-five patients $(66 \%)$ had good 
performance status (ECOG performance status; $0-1)$. Twenty-six patients (31\%) had liver metastases and 30 patients $(36 \%)$ had ascites resulting from peritoneal dissemination.

Of the 83 patients, the NLRs at 1 st-line initiation ranged from 0.72 to 48.9 (the median NLR was 3.00). Based on the definitive cut-off value for NLR, 42 patients were categorized into an NLR-low group (NLR < 3.00) and 41 patients into an NLR-high group (NLR $\geq 3.00$, Fig. 1).

At the time of the analysis, 12 patients were still undergoing 1st-line treatment, 15 patients had been transferred to best supportive care (BSC) after 1 st-line treatment, and 56 patients (79\%) had received 2ndline treatment. Of the 56 patients, 38 patients were classified into the NLR-low group and 18 patients into the NLR-high group according to their blood NLRs evaluated prior to the start of 2nd-line treatment.

Among the 56 2nd-line treatment patients, 4 were still undergoing 2nd-line treatment and 34 (65\%) had received 3rd-line treatment. Of the 34 3rd-line treatment patients, 23 were categorized into the NLR-low group and 11 patients into the NLR-high group according to blood NLR values prior to 3rd-line treatment. Finally, of the 34 patients treated with 3rd-line chemotherapy, 2 were still under 3rd-line treatment, 21 $(66 \%)$ received 4 th-line chemotherapy, and the remaining 11 patients were transferred to BSC.

\section{Clinical outcome in relation to NLR values prior to each chemotherapy line}

Table 2 presents clinicopathological characteristics of GC patients at each chemotherapy line in relation to the NLR-high and NLR-low groups. Regarding the patient characteristics, the NLR-high group had significantly more patients with a poor performance status and ascites throughout the course of chemotherapy.

Of the 83 patients who were analyzed, the median OS was 13.2 months ( $95 \% \mathrm{Cl}$ : $9.1-17.9)$. When we divided the patients into two groups based on NLR value evaluated prior to 1st-line chemotherapy, the median OS was shorter in the NLR-high group compared with the NLR-low group (OS: 8.0 months [95\% Cl: 5.7-13.2] vs. 18.1 months [11.9-20.9], $p=0.06$, Fig.2, Panel A). Of 56 patients who received 2nd-line chemotherapy, the median OS was significantly shorter in the NLR-high group compared with the NLR-low group (OS: 4.5 months [95\% Cl: 3.4-6.0] vs. 10.7 months [95\% Cl: 7.4-13.7], $p<0.05$, Fig.2, Panel B). Of the 34 patients who received 3rd-line chemotherapy, the median OS was significantly shorter in the NLRhigh group compared with the NLR-low group (OS: 4.7 months [ $95 \% \mathrm{Cl}: 0.8-7.3]$ vs. 8.7 months [95\% Cl: 5.6-14.0], $p<0.05$, Fig.2, Panel C).

Next, we focused on the 24 patients who received nivolumab monotherapy during 3rd-line treatment. According to the NLR value prior to nivolumab therapy, 14 patients $(55 \%)$ were stratified into the NLR-low group and 10 patients into the NLR-high group. Patient characteristics are shown in Table 3. The median OS was shorter in the NLR-high group compared with that in the NLR-low group (median OS: 6.6 months [95\% Cl: 0.8-7.3 months] vs. 8.3 months [2.6-10.3 months], $p=0.06$, Fig.2, Panel D).

Changes in NLR value throughout chemotherapeutic drug treatment in unresectable GC patients 
We examined fluctuations of pretreatment NLR values in each case throughout the course of chemotherapy (Fig. 3). Among 35 NLR-low patients at 1 st-line who were eligible for 2 nd-line treatment and categorized into the NLR-low group by pretreatment NLR values at 1 st-line, $23(66 \%)$ retained their NLR within 3.0 and only 4 cases (13\%) showed an increase in NLR by 3.0 or more prior to 2 nd-line therapy. In contrast, of the $35 \mathrm{NLR}$-high patients at 1 st-line who were transferred to 2 nd-line therapy, although 15 patients (43\%) recovered, their NLR was within 3.0 , and $14(40 \%)$ retained an NLR 3.0 value or more prior to 2 nd-line therapy ( $p=0.026$, Fig. 4 ).

\section{Discussion}

In this study, we revealed that the NLR value in blood obtained before initiation of 1 st-, 2 nd-, and 3rd-line chemotherapy represents a prognostic biomarker for unresectable GC. Recently, several predictive and prognostic factors were evaluated in various cancer types to accurately define patient groups that may benefit from anticancer therapy and predict their survival. As systemic inflammation plays an important role in tumor promotion and progression (7), systemic inflammatory markers including CRP, PLR, and NLR, have attracted attention as putative prognostic markers in various cancer types (10-12). Peripheral blood NLR, an indicator of systemic inflammation, is a simple and conventional biomarker that has been considered a prognostic factor in multiple solid neoplasms including $\operatorname{GC}(9,16)$. According to a recent report by Zhou et al., pretreatment NLR was a significant independent prognostic factor for progressionfree survival (PFS) and OS, and elevated NLR was associated with poor PFS and OS in unresectable GC patients treated with first-line chemotherapy (17).

In a series of studies that examined prognostic and predictive effects of NLR in cancer patients, NLR was measured before the initiation of first-line chemotherapy or treatment $(17,18)$. Obviously, NLRs are not fixed values and fluctuate with patient conditions. Therefore, we examined whether it could predict clinical outcomes when the NLR values were determined prior to initiation of each line of palliative chemotherapy. Our results demonstrate reproducibly that pretreatment NLR values for each chemotherapy line can predict clinical outcome for unresectable GC patients.

Consistent with previous studies, we evaluated whether NLR values obtained before initiation of 1 st-line chemotherapy could predict clinical outcome in 2nd-line and 3rd-line treatment. However, the groups divided by NLR value obtained before 1st-line chemotherapy did not show the expected clinical outcomes in 2 nd- and 3rd-line treatment. This may be partially explained by the data we presented in Figures 3 and 4. Approximately, $66 \%$ of NLR-low patients who were transferred from 1 st- to 2 nd-line chemotherapy retained their NLR value within 3.0. However, it was rare that patients had increased NLR above 3.0 at pretreatment of 2 nd-line therapy. In contrast, although $43 \%$ of NLR-high patients retained NLR values within 3.0 at pretreatment of 2nd-line therapy, 40\% of NLR-high patients retained their NLR 3.0 or more at pretreatment of 3rd-line therapy. Thus, patients with lower NLR values at pretreatment of 1 st-line therapy were in good condition throughout their treatment. Only a few patients with higher NLR values at pretreatment of 1 st-line therapy retained their clinical condition throughout chemotherapy. 
Wang et al. reported that the OS of unresectable GC patients whose NLR levels increased after 1st-line treatment was 9 months, whereas that of patients with decreased NLR was 20 months (19). Changes in NLR following chemotherapy was reported to predict prognosis in multiple carcinomas (19-21). Our results also demonstrate that the mean NLR value significantly decreased in patients who were transferred to 2nd-line treatment, whereas it increased in patients who could not be transferred to 2 nd-line treatment.

Next, we focused on the association of pretreatment NLR with clinical outcome of 3rd-line nivolumab monotherapy patients. Nivolumab, a PD-1 inhibitor, was evaluated in a randomized phase III study in GC patients treated with $\geq 2$ prior chemotherapy regimens and significantly prolonged oS compared with the placebo group (5). However, approximately half of the patients treated with nivolumab did not receive a survival benefit compared with the placebo (22). In recent years, numerous biomarkers, including tumor genomic markers, neoantigens, the tumor immune microenvironment phenotype, and liquid biopsy markers, have been evaluated in association with immune checkpoint inhibitors. Kumagai et al. showed that, in cancer patients including GC, the frequency of PD-1+CD8+T cells relative to that of PD-1+ regulatory $\mathrm{T}$ cells in the tumor microenvironment predict clinical outcome of PD-1 blockade therapies and is superior to other biomarkers, including PD-L1 expression or tumor mutational burden (23). However, these biomarkers are often difficult to measure and introduce in clinical practice. In contrast, the advantage of peripheral blood NLR is that it is routinely measured in cancer patients, so this parameter is readily available to physicians. Ogata et al. reported that in 26 advanced GC patients treated with nivolumab, the median OS was significantly longer in GC patients with lower NLR values (24). Pretreatment NLR of patients treated with 3rd-line nivolumab may be a predictor of OS. Similarity, in our analysis of patients treated with 3rd-line nivolumab monotherapy, pretreatment NLR before nivolumab induction could predict the prognosis of unresectable GC patients.

This study has some limitations. It is a single-center, retrospective study with a relatively small sample size. Notwithstanding, the clinical outcomes results are similar to that typically reported for unresectable GC. However, for the first time, we identified an association with NLR and clinical outcome in each chemotherapy line as well as 3rd-line nivolumab monotherapy. To confirm our results, a future prospective analysis will be required.

\section{Conclusions}

The pretreatment NLR obtained prior to the initiation of each chemotherapy line may predict clinical outcome for unresectable GC patients. Particularly, as NLRs are not fixed values, but fluctuate with patient conditions, it is reasonable to evaluate NLR values in blood before the initiation of each line of chemotherapy to accurately predict prognosis.

\section{List Of Abbreviations}

BSC: best supportive care 
CRP: $c$ reactive protein

GC: gastric cancer

ICl: immune checkpoint inhibitor

NLR: neutrophil-to-lymphocyte ratio

OS: overall survival

PD-1: program death-1

PD-L1: program death ligand-1

PFS: progression-free survival

PLR: platelet-lymphocyte ratio

\section{Declarations}

\section{Ethics approval and consent to participate}

This study was approved by the Institutional Review Board of Kawasaki Medical University Hospital (IRB number: 3939-01).

\section{Consent for publication}

Not applicable.

\section{Availability of data and materials}

The datasets used and/or analyzed during the current study are available from the corresponding author upon reasonable request.

\section{Competing interests}

The authors declare that they have no competing interests.

\section{Funding}

This study was supported in part by a Research Project Grant (R01B066 and R02B048) from Kawasaki Medical School.

\section{Author contributions}

HT performed all analyses and drafted the manuscript. SY, MO and YK performed the patient care and summarized the clinicopathological data. TY, AN, and KT assisted with the data interpretation. TN 
designed the project, performed the analyses, assisted with the interpretation of all data, secured funding, and drafted the manuscript. All authors read and approved the final manuscript.

\section{Acknowledgments}

The authors would like to thank Dr. HarukoTanioka for technical assistance with the experiments.

We are also grateful to the referees for useful comments.

\section{References}

1. Ferlay J, Soerjomataram I, Dikshit R, Eser S, Mathers C, Rebelo M, et al. Cancer incidence and mortality worldwide: sources, methods and major patterns in GLOBOCAN 2012. International journal of cancer. 2015;136(5):E359-86.

2. Glimelius B, Ekström K, Hoffman K, Graf W, Sjödén PO, Haglund U, et al. Randomized comparison between chemotherapy plus best supportive care with best supportive care in advanced gastric cancer / ESMO. Annals of oncology: official journal of the European Society for Medical Oncology. 1997;8(2):163-8.

3. Thuss-Patience PC, Kretzschmar A, Bichev D, Deist T, Hinke A, Breithaupt K, et al. Survival advantage for irinotecan versus best supportive care as second-line chemotherapy in gastric cancer-a randomised phase III study of the Arbeitsgemeinschaft Internistische Onkologie (AIO). European journal of cancer (Oxford, England: 2011);47(15):2306-14.

4. Kang JH, Lee SI, Lim DH, Park KW, Oh SY, Kwon HC, et al. Salvage chemotherapy for pretreated gastric cancer: a randomized phase III trial comparing chemotherapy plus best supportive care with best supportive care alone. Journal of clinical oncology: official journal of the American Society of Clinical Oncology. 2012;30(13):1513-8.

5. Kang YK, Boku N, Satoh T, Ryu MH, Chao Y, Kato K, et al. Nivolumab in patients with advanced gastric or gastro-oesophageal junction cancer refractory to, or intolerant of, at least two previous chemotherapy regimens (ONO-4538-12, ATTRACTION-2): a randomised, double-blind, placebocontrolled, phase 3 trial. Lancet. 2017;390(10111):2461-71.

6. Yamada Y, Higuchi K, Nishikawa K, Gotoh M, Fuse N, Sugimoto N, et al. Phase III study comparing oxaliplatin plus S-1 with cisplatin plus S-1 in chemotherapy-naive patients with advanced gastric cancer / ESMO. Annals of oncology: official journal of the European Society for Medical Oncology. 2015;26(1):141-8.

7. Hanahan D, Weinberg RA. Hallmarks of cancer: the next generation. Cell. 2011;144(5):646-74.

8. Diem S, Schmid S, Krapf M, Flatz L, Born D, Jochum W, et al. Neutrophil-to-lymphocyte ratio (NLR) and Platelet-to-Lymphocyte ratio (PLR) as prognostic markers in patients with non-small cell lung cancer (NSCLC) treated with nivolumab. Lung cancer. 2017;111:176-81.

9. Murakami Y, Saito H, Shimizu S, Kono Y, Shishido Y, Miyatani K, et al. Neutrophil-to-lymphocyte ratio as a prognostic indicator in patients with unresectable gastric cancer. Anticancer research. 
2019;39(5):2583-9.

10. Ethier JL, Desautels D, Templeton A, Shah PS, Amir E. Prognostic role of neutrophil-to-lymphocyte ratio in breast cancer: a systematic review and meta-analysis. Breast cancer research: BCR. 2017;19(1):2.

11. Iwai N, Okuda T, Sakagami J, Harada T, Ohara T, Taniguchi M, et al. Neutrophil to lymphocyte ratio predicts prognosis in unresectable pancreatic cancer. Scientific reports. 2020;10(1):18758.

12. Ciocan A, Bolboacă SD, Drugan C, Ciocan RA, Graur F, Al Hajjar N. The Pattern of calculated inflammation ratios as prognostic values in patients with colorectal cancer. Combinatorial chemistry and high throughput screening. 2021;24(9):1428-35.

13. Bagley SJ, Kothari S, Aggarwal C, Bauml JM, Alley EW, Evans TL, et al. Pretreatment neutrophil-tolymphocyte ratio as a marker of outcomes in nivolumab-treated patients with advanced non-smallcell lung cancer. Lung cancer. 2017;106:1-7.

14. Caziuc A, Schlanger D, Amarinei G, Dindelegan GC. Neutrophils-to-lymphocytes, lymphocytes tomonocytes and platelets-to-lymphocytes ratios - predictive biomarkers for response to neoadjuvant chemotherapy in breast cancer. Journal of B.U.ON.: official journal of the Balkan Union of Oncology. 2020;25(1):182-7.

15. Valero C, Lee M, Hoen D, Weiss K, Kelly DW, Adusumilli PS, et al. Pretreatment neutrophil-tolymphocyte ratio and mutational burden as biomarkers of tumor response to immune checkpoint inhibitors. Nature communications. 2021;12(1):729.

16. Ock CY, Nam AR, Lee J, Bang JH, Lee KH, Han SW, et al. Prognostic implication of antitumor immunity measured by the neutrophil-lymphocyte ratio and serum cytokines and angiogenic factors in gastric cancer. Gastric cancer: official journal of the International Gastric Cancer Association and the Japanese Gastric Cancer Association. 2017;20(2):254-62.

17. Zhou D, Wu Y, Zhu Y, Lin Z, Yu D, Zhang T. The prognostic value of neutrophil-to-lymphocyte ratio and monocyte-to-lymphocyte ratio in metastatic gastric cancer treated with systemic chemotherapy. Journal of cancer. 2020;11(14):4205-12.

18. Liu H, Song M, Fang F, Gao X, Zhang Z, Wang S. Prediction of chemotherapeutic efficacy using the ratio of neutrophils to lymphocytes in patients with unresectable or recurrent gastric cancer. Oncology letters. 2015;10(4):2244-8.

19. Wang F, Liu ZY, Xia YY, Zhou C, Shen XM, Li XL, et al. Changes in neutrophil/lymphocyte and platelet/lymphocyte ratios after chemotherapy correlate with chemotherapy response and prediction of prognosis in patients with unresectable gastric cancer. Oncology letters. 2015;10(6):3411-8.

20. McLellan P, Henriques J, Ksontini F, Doat S, Hammel P, Desrame J, et al. Prognostic value of the early change in neutrophil-to-lymphocyte ratio in metastatic pancreatic adenocarcinoma. Clinics and research in hepatology and gastroenterology. 2021;45(3):101541.

21. Kim JY, Jung EJ, Kim JM, Lee HS, Kwag SJ, Park JH, et al. Dynamic changes of neutrophil-tolymphocyte ratio and platelet-to-lymphocyte ratio predicts breast cancer prognosis. BMC cancer. 2020;20(1):1206. 
22. Chen LT, Satoh T, Ryu MH, Chao Y, Kato K, Chung HC, et al. A phase 3 study of nivolumab in previously treated advanced gastric or gastroesophageal junction cancer (ATTRACTION-2): 2-year update data. Gastric cancer: official journal of the International Gastric Cancer Association and the Japanese Gastric Cancer Association. 2020;23(3):510-9.

23. Kumagai S, Togashi Y, Kamada T, Sugiyama E, Nishinakamura H, Takeuchi Y, et al. The PD-1 expression balance between effector and regulatory $T$ cells predicts the clinical efficacy of PD-1 blockade therapies. Nature immunology. 2020;21(11):1346-58.

24. Ogata T, Satake H, Ogata M, Hatachi Y, Inoue K, Hamada M, Yasui H. Neutrophil-to-lymphocyte ratio as a predictive or prognostic factor for gastric cancer treated with nivolumab: a multicenter retrospective study. Oncotarget. 2018;9(77):34520-7.

\section{Tables}

Due to technical limitations, table 1 to 3 PDFs are only available as a download in the Supplemental Files section.

\section{Figures}

\section{Figure1}

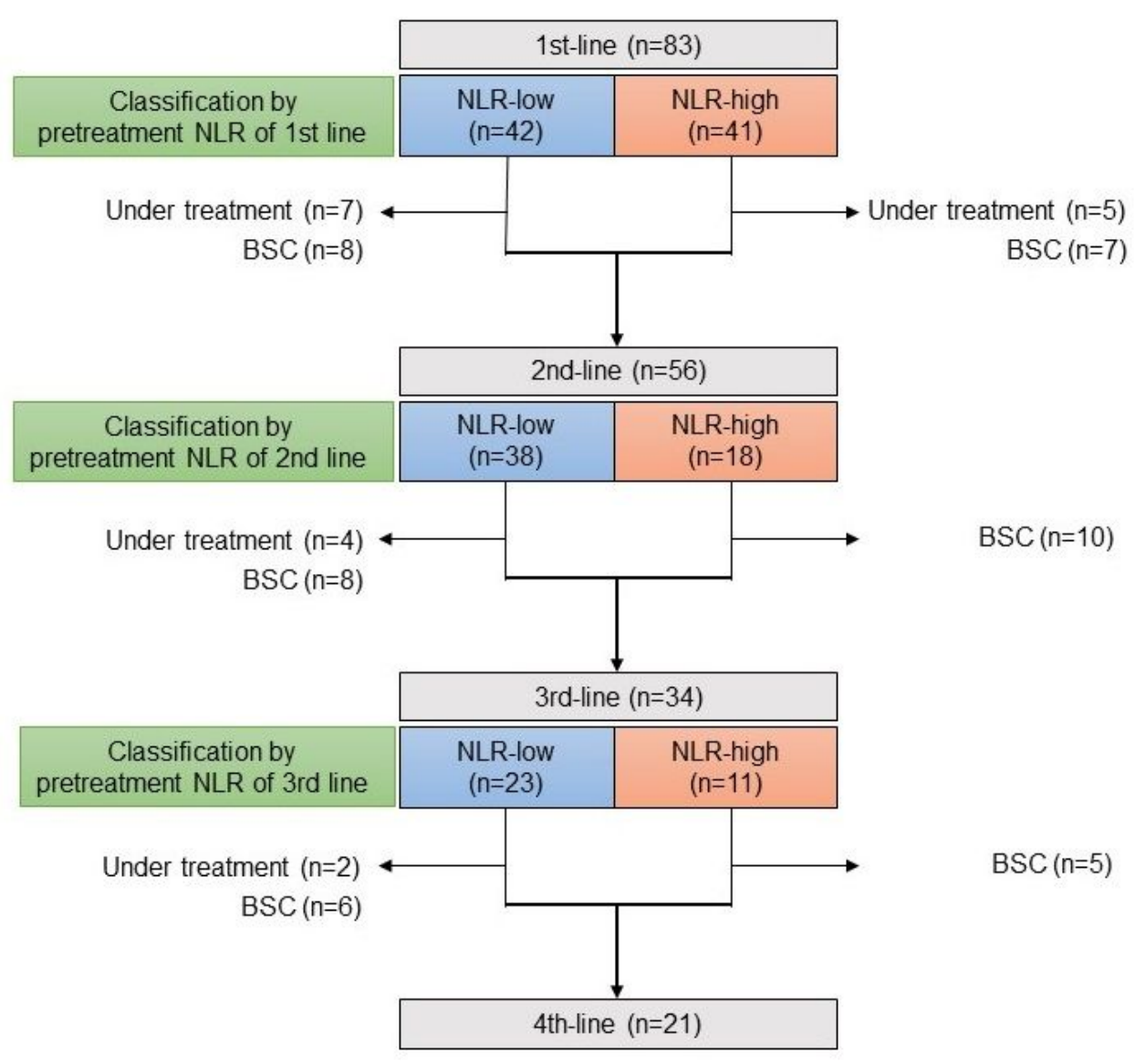


Figure 1

The STROBE diagram of the GC patient cohort. Treatment progress according to classification by pretreatment NLR for each line.
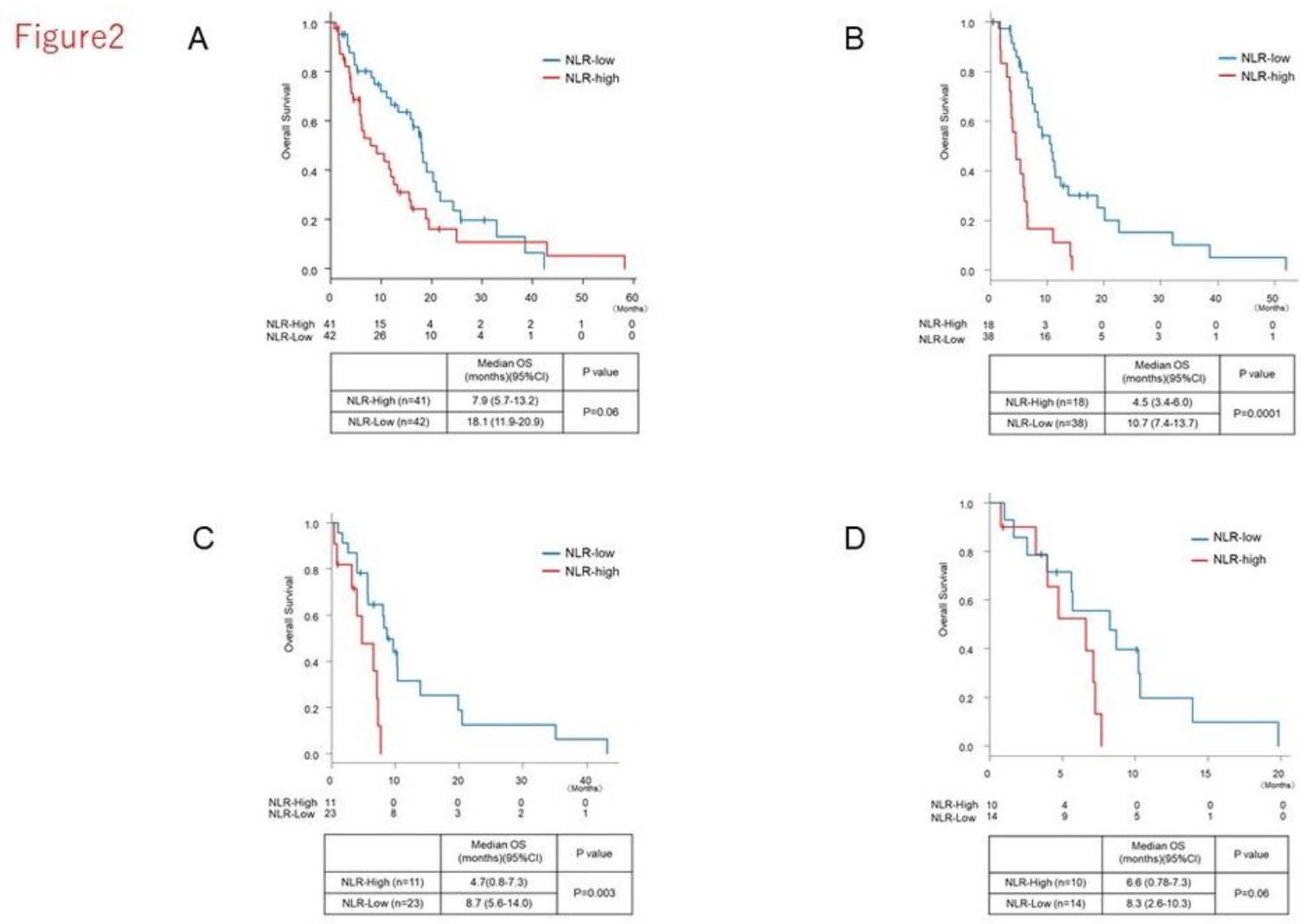

\section{Figure 2}

Kaplan-Meier curves of OS according to NLR. Kaplan-Meier analysis for 1st-line OS according to 1st-line pretreatment NLR (A), for 2nd-line OS according to 2nd-line pretreatment NLR (B) and for 3rd-line OS according to 3rd-line pretreatment NLR (C). Kaplan-Meier curves for OS of patients who received nivolumab monotherapy for 3rd-line chemotherapy according to NLR (D). 

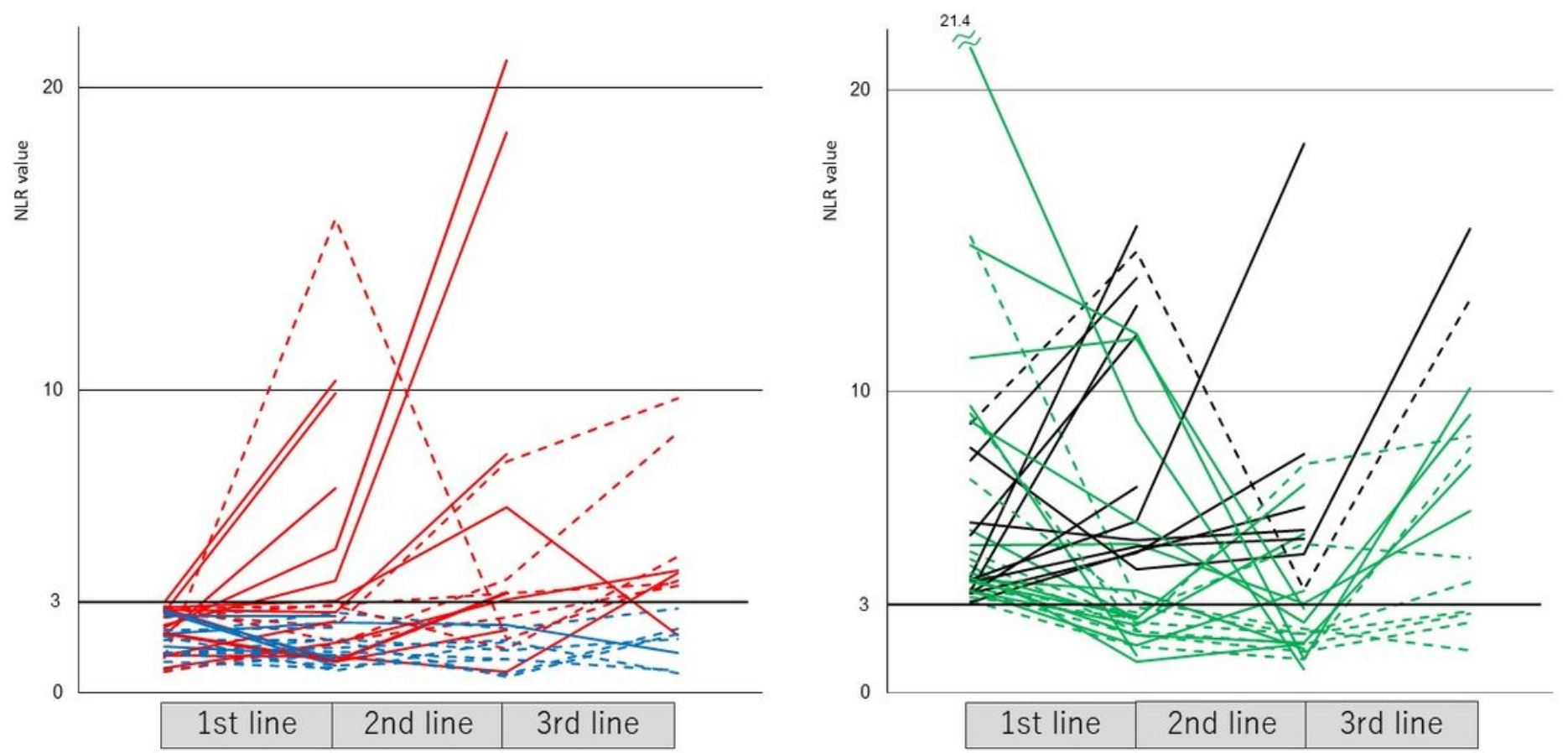

\section{Figure 3}

Changes of the NLR value throughout chemotherapy treatment in unresectable GC patients. Fluctuations of pretreatment NLR values in each case were categorized into an NLR-low group (A) and an NLR-high group (B) by 1st-line pretreatment NLR value. Dotted lines denote the patients under treatment at the time of the analysis. Solid lines denote the patients who had no chemotherapy treatment at the time of the analysis. A red dot or solid line denotes the patients who had a worse NLR value (>3.0) during chemotherapy. A blue dot or solid line denotes the patients who had a lower NLR value $(<3.0)$ throughout chemotherapy. A green dot or solid line denotes the patients who had a lower NLR value $(<3.0)$ during chemotherapy at least once. A black dot or solid line denotes the patients who had high NLR values (> 3.0) throughout chemotherapy. 


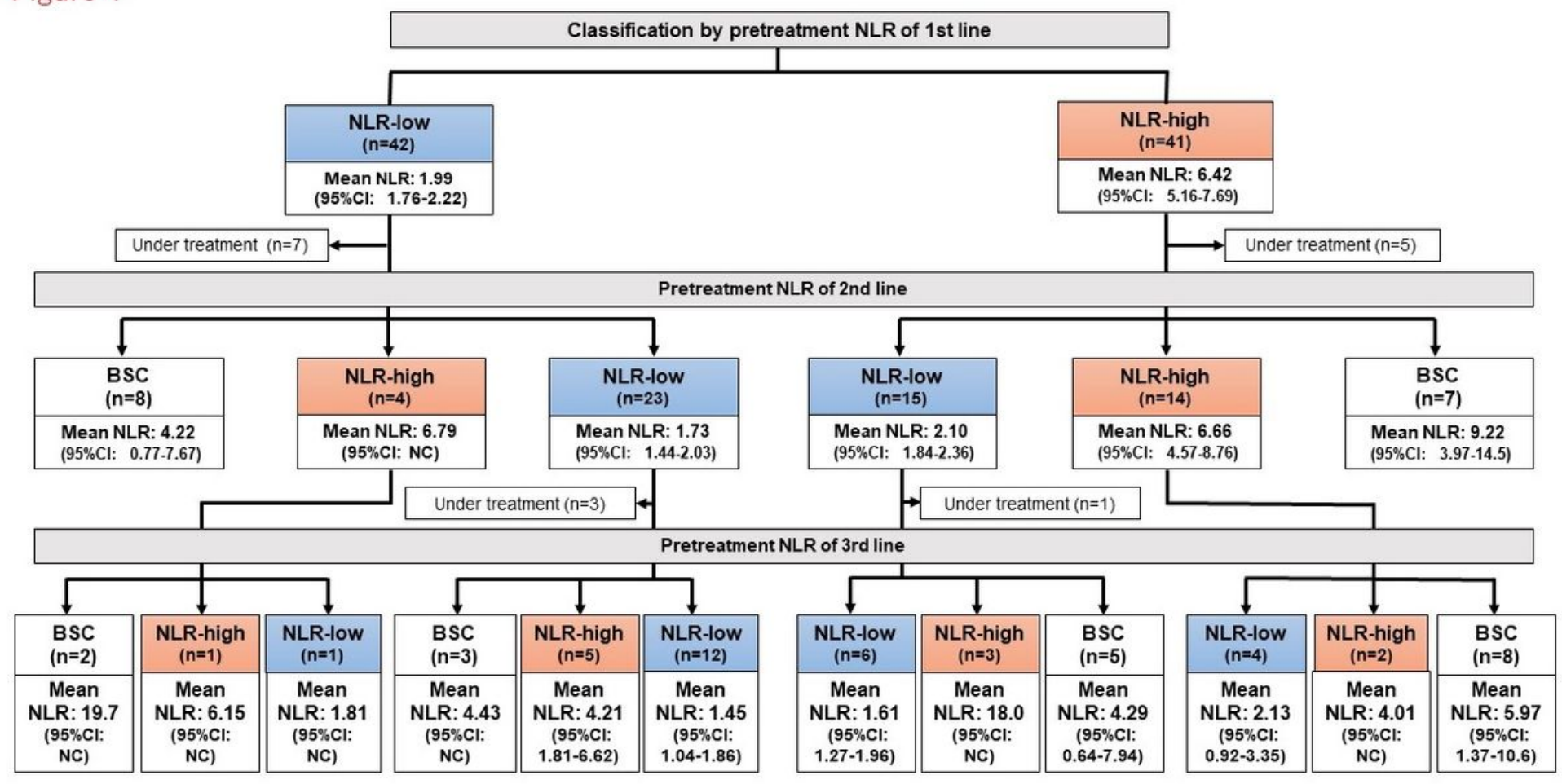

\section{Figure 4}

Precise treatment progress according to classification by pretreatment NLR for each line. Mean NLR value and $95 \% \mathrm{Cl}$ are listed under the column. NC denotes not calculated.

\section{Supplementary Files}

This is a list of supplementary files associated with this preprint. Click to download.

- Table1.pdf

- Table2.pdf

- Table3.pdf 